\title{
BSM physics at CLIC
}

\author{
Rosa Simoniello*广 \\ CERN \\ E-mail: rosa.simoniello@cern.ch
}

The Compact Linear Collider (CLIC) is an option for a future electron-positron collider operating at centre-of-mass energies from a few hundred $\mathrm{GeV}$ up to $3 \mathrm{TeV}$. The search for phenomena beyond the Standard Model through direct observation of new particles and precision measurements is one of the main motivations for the high-energy stages of CLIC. An overview of physics benchmark studies assuming different new physics scenarios is given in this contribution. These studies are based on full detector simulations. New particles can be discovered in most of the considered scenarios almost up to the kinematic limit ( $\sqrt{s} / 2$ for pair production). The low background conditions at CLIC provide extended discovery potential compared to hadron colliders, for example in the case of non-coloured $\mathrm{TeV}$-scale SUSY particles. In addition to direct particle searches, BSM models can be probed up to scales of tens of $\mathrm{TeV}$ through precision measurements. Examples, including recent results on the reaction $e^{+} e^{-} \rightarrow \gamma \gamma$, are given. Beam polarisation allows to constrain the underlying theory further in many cases. The discussion of LHC results relevant for the CLIC physics case is also included.

38th International Conference on High Energy Physics 3-10 August 2016

Chicago, USA
${ }^{*}$ Speaker.
${ }^{\dagger}$ On behalf of the CLICdp collaboration 


\section{Introduction}

The Compact Linear Collider (CLIC) is a proposed high-energy and high-luminosity $e^{+} e^{-}$ linear collider with a rich physics program and high potential for discovery of new physics. It is planned to be built in three stages with centre-of-mass energy of $380 \mathrm{GeV}, 1.5 \mathrm{TeV}$ and $3 \mathrm{TeV}$ [1]. The first energy stage is devoted to precision measurements of Higgs and top properties, while the high-energy stages aim to beyond Standard Model (BSM) and rare Higgs processes.

This compelling physics program puts strict requirements on the detector design and technology, resulting in an ambitious R\&D program. The detector models used for the following studies are based on the ILD [2] and SiD [3] detector concepts at the International Linear Collider (ILC) [4] adapted for the CLIC beam conditions at $3 \mathrm{TeV}$. The key elements include an ultra-light vertex detector with excellent $b$-physics capabilities, highly-granular calorimeters designed for particle flow techniques, and high forward coverage for electromagnetic objects down to $10 \mathrm{mrad}$ [5].

Except when stated differently, all the following studies are carried out using a full-detector simulation with the main induced background, $\gamma \gamma \rightarrow$ hadrons, overlaid. On the other hand, beam polarisation ( $80 \%$ for the electron beam, $0 \%$ for the positron beam) is assumed for the analysis only when explicitly stated.

\section{BSM program at CLIC}

CLIC offers an exciting possibility to search for new physics beyond the Standard Model. Lepton collisions would thus allow to explore different processes and production mechanisms with respect to hadron colliders, extending and complementing the LHC program.

The BSM program at CLIC is divided into direct and indirect searches. Direct searches for possible new particles production profit from the high centre-of-mass energy available. Indirect searches focus on precision measurements of some SM parameters: the clean environment and the low level of background provided by lepton collisions will allow to improve the precision on many measurements beyond the LHC reach. Deviations from SM predictions would be interpreted as signs of new physics and could probe BSM theories up to tens of TeV, well beyond the spectra of particles that could be directly produced.

\section{Direct searches}

Direct searches at CLIC mostly focus on Supersymmetry (SUSY) benchmark models [5, 6] to prove the potential physics reach of the experiment and to demonstrate key aspects of the detector performance. The chosen SUSY models are marked as $I, I I, I I I$ and the respective production cross sections are shown in Figure 1. The spectra of particles for these models include sleptons, squarks and heavy Higgses, for which the LHC limits are not very stringent. Moreover, given the wide range of mass and spin for the predicted particles, the validity of these studies can be extended from SUSY to any other theory interpretation that predicts particles with the same quantum numbers.

If any new particle is discovered, either at LHC or at CLIC itself, CLIC could provide precise measurements of the properties of such a particle, like mass, couplings and spin. In general, a precision of $\mathrm{O}(1 \%)$ is found on the mass measurement of different sparticles across different channels and SUSY models. The full list of results is reported in Table 1. 

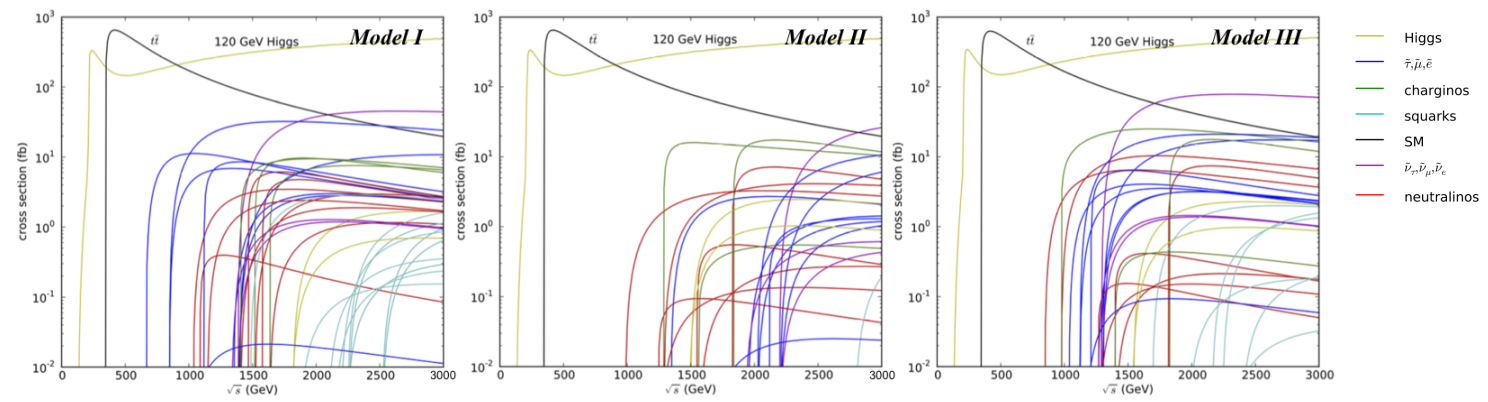

Figure 1: The production cross sections as a function of the centre-of-mass energy for the SUSY Model $I$, $I I$ and $I I I$, respectively from left to right [6].

\begin{tabular}{|c|c|c|c|c|c|c|}
\hline $\begin{array}{l}\sqrt{s} \\
(\mathrm{TeV})\end{array}$ & Particles & Decay mode & $\begin{array}{l}\text { SUSY } \\
\text { model }\end{array}$ & $\begin{array}{l}\text { Measured } \\
\text { quantity }\end{array}$ & $\begin{array}{l}\text { Generator } \\
\text { value }(\mathrm{GeV})\end{array}$ & $\begin{array}{l}\text { Stat. } \\
\text { uncert. }\end{array}$ \\
\hline \multirow{6}{*}{3.0} & \multirow{6}{*}{ Sleptons } & \multirow{2}{*}{$\widetilde{\mu}_{\mathrm{R}}^{+} \widetilde{\mu}_{\mathrm{R}}^{-} \rightarrow \mu^{+} \mu^{-} \widetilde{\chi}_{1}^{0} \widetilde{\chi}_{1}^{0}$} & \multirow{6}{*}{ II } & $\tilde{\ell}$ mass & 1010.8 & $0.6 \%$ \\
\hline & & & & \multirow{5}{*}{$\begin{array}{l}\tilde{\chi}_{1}^{0} \text { mass } \\
\tilde{\ell} \text { mass }\end{array}$} & 340.3 & $1.9 \%$ \\
\hline & & \multirow{2}{*}{$\widetilde{\mathrm{e}}_{\mathrm{R}}^{+} \widetilde{\mathrm{e}}_{\mathrm{R}}^{-} \rightarrow \mathrm{e}^{+} \mathrm{e}^{-} \widetilde{\chi}_{1}^{0} \widetilde{\chi}_{1}^{0}$} & & & 1010.8 & $0.3 \%$ \\
\hline & & & & & 340.3 & $1.0 \%$ \\
\hline & & \multirow{2}{*}{$\widetilde{v}_{\mathrm{e}} \widetilde{\mathrm{v}}_{\mathrm{e}} \rightarrow \widetilde{\chi}_{1}^{0} \widetilde{\chi}_{1}^{0} \mathrm{e}^{+} \mathrm{e}^{-} \mathrm{W}^{+} \mathrm{W}^{-}$} & & & 1097.2 & $0.4 \%$ \\
\hline & & & & & 643.2 & $0.6 \%$ \\
\hline \multirow{2}{*}{3.0} & Chargino & $\tilde{\chi}_{1}^{+} \widetilde{\chi}_{1}^{-} \rightarrow \tilde{\chi}_{1}^{0} \tilde{\chi}_{1}^{0} \mathrm{~W}^{+} \mathrm{W}^{-}$ & \multirow{2}{*}{ II } & $\widetilde{\chi}_{1}^{ \pm}$mass & 643.2 & $1.1 \%$ \\
\hline & Neutralino & $\widetilde{\chi}_{2}^{0} \widetilde{\chi}_{2}^{0} \rightarrow \mathrm{h} / \mathrm{Z}^{0} \mathrm{~h} / \mathrm{Z}^{0} \widetilde{\chi}_{1}^{0} \widetilde{\chi}_{1}^{0}$ & & $\tilde{\chi}_{2}^{0}$ mass & 643.1 & $1.5 \%$ \\
\hline 3.0 & Squarks & $\widetilde{\mathrm{q}}_{\mathrm{R}} \widetilde{\mathrm{q}}_{\mathrm{R}} \rightarrow \mathrm{q} \overline{\mathrm{q}} \widetilde{\chi}_{1}^{0} \widetilde{\chi}_{1}^{0}$ & $\mathrm{I}$ & $\widetilde{\mathrm{q}}_{\mathrm{R}}$ mass & 1123.7 & $0.5 \%$ \\
\hline \multirow{2}{*}{3.0} & \multirow{2}{*}{ Heavy Higgs } & $\mathrm{H}^{0} \mathrm{~A}^{0} \rightarrow \mathrm{b} \overline{\mathrm{b}} \mathrm{b} \overline{\mathrm{b}}$ & \multirow{2}{*}{ I } & $\mathrm{H}^{0} / \mathrm{A}^{0}$ mass & $902.4 / 902.6$ & $0.3 \%$ \\
\hline & & $\mathrm{H}^{+} \mathrm{H}^{-} \rightarrow \mathrm{tb} \bar{b} \overline{\mathrm{t}}$ & & $\mathrm{H}^{ \pm}$mass & 906.3 & $0.3 \%$ \\
\hline \multirow{6}{*}{1.4} & \multirow{6}{*}{ Sleptons } & $\tilde{\mu}^{+} \tilde{\mu}^{-} \rightarrow u^{+} \mu^{-} \widetilde{\gamma}^{0} \widetilde{\gamma}^{0}$ & \multirow{6}{*}{ III } & $\widetilde{\ell}$ mass & 560.8 & $0.1 \%$ \\
\hline & & $\mu_{\mathrm{R}} \mu_{\mathrm{R}} \rightarrow \mu \mu \chi_{1} \chi_{1}$ & & ${\underset{\sim}{\alpha}}_{1}^{0}$ mass & 357.8 & $0.1 \%$ \\
\hline & & $\widetilde{\gamma}_{0}^{0} 00$ & & $\tilde{\ell}$ mass & 558.1 & $0.1 \%$ \\
\hline & & $e_{R} e_{R}$ & & $\tilde{\chi}_{1}^{0}$ mass & 357.1 & $0.1 \%$ \\
\hline & & \multirow{2}{*}{$\widetilde{v}_{\mathrm{e}} \widetilde{v}_{\mathrm{e}} \rightarrow \widetilde{\chi}_{1}^{0} \widetilde{\chi}_{1}^{0} \mathrm{e}^{+} \mathrm{e}^{-} \mathrm{W}^{+} \mathrm{W}^{-}$} & & $\tilde{\ell}$ mass & 644.3 & $2.5 \%$ \\
\hline & & & & $\tilde{\chi}_{1}^{ \pm}$mass & 487.6 & $2.7 \%$ \\
\hline 1.4 & Stau & $\tilde{\tau}_{1}^{+} \widetilde{\tau}_{1}^{-} \rightarrow \tau^{+} \tau^{-} \widetilde{\chi}_{1}^{0} \widetilde{\chi}_{1}^{0}$ & III & $\tilde{\tau}_{1}$ mass & 517 & $2.0 \%$ \\
\hline \multirow{2}{*}{1.4} & Chargino & $\tilde{\chi}_{1}^{+} \widetilde{\chi}_{1}^{-} \rightarrow \widetilde{\chi}_{1}^{0} \widetilde{\chi}_{1}^{0} \mathrm{~W}^{+} \mathrm{W}^{-}$ & \multirow{2}{*}{ III } & $\tilde{\chi}_{1}^{ \pm}$mass & 487 & $0.2 \%$ \\
\hline & Neutralino & $\widetilde{\chi}_{2}^{0} \widetilde{\chi}_{2}^{0} \rightarrow \mathrm{h} / \mathrm{Z}^{0} \mathrm{~h} / \mathrm{Z}^{0} \widetilde{\chi}_{1}^{0} \widetilde{\chi}_{1}^{0}$ & & $\tilde{\chi}_{2}^{0}$ mass & 487 & $0.1 \%$ \\
\hline
\end{tabular}

Table 1: Summary of the CLIC SUSY benchmark analyses results obtained with full-detector simulation with background overlaid [6].

Another very interesting search at CLIC is the direct production of Dark Matter (DM). The presence of DM is now well established but very little is known about its nature. The analysis, at CLIC is based on the WIMP paradigm (weakly interacting massive particles) that assumes a weak ${ }^{1}$ interaction between DM and SM particles. The most promising channel at CLIC is the mono$\gamma+p_{\mathrm{T}}^{\text {miss }}$ final state. For this analysis the identification of very forward electrons and photons has proven to be essential, therefore studies are ongoing to reduce the associated systematic errors.

\footnotetext{
${ }^{1}$ Not necessarily coming from the weak nuclear force.
} 


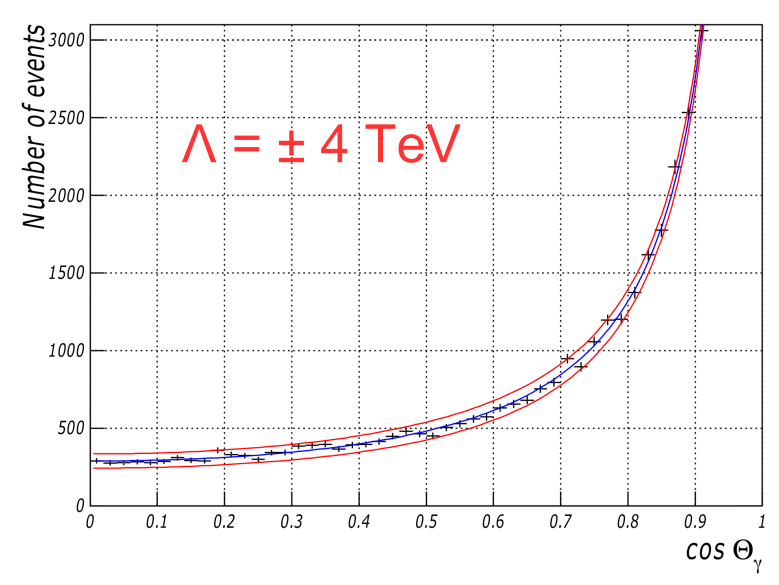

Figure 2: The expected $\gamma \gamma$ angular distribution for the SM (blue) and $\Lambda= \pm 4 \mathrm{TeV}$ (red) [7].

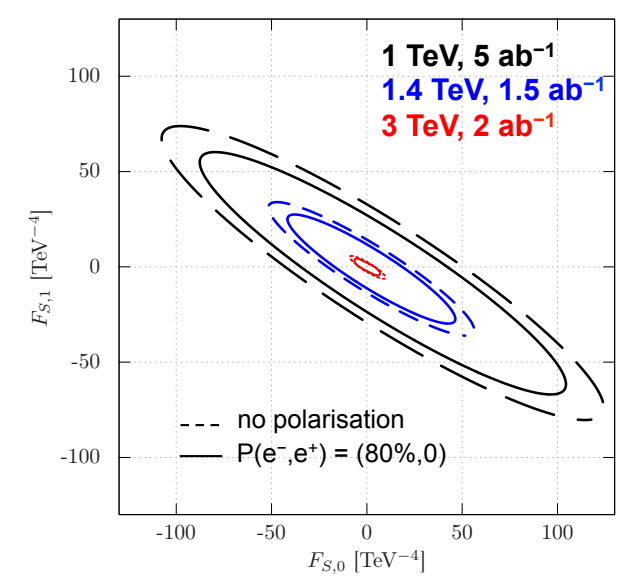

Figure 3: Precision on the $F_{S 0}, F_{S 1}$ couplings for different $\sqrt{s}$ and polarisations [9].

\section{Indirect searches}

CLIC is very well suited for precision studies with a rich program covering many different areas. Extensive tests of the SM with improved accuracy will hopefully allow to see new phenomena.

Possible extensions to the QED theory, like finite electron size, extra dimensions and excited electrons, can be tested through the measurement of the $e e \rightarrow \gamma \gamma$ cross section and the angular distribution of the $\gamma \gamma$ system [7]. For example, in the finite electron size theory, the cross section for the process can be written as the Born cross section with an additional term regulated by an energy cut off, $\Lambda$, that can then be translated into the actual size of the electron. Figure 2 shows the expected $\gamma \gamma$ angular distribution for the SM and for $\Lambda=4 \mathrm{TeV}$. Beamstrahlung and initial state radiation could distort the angular distribution of the $\gamma \gamma$ system, therefore, only events where two high energetic photons are back-to-back are selected. The main backgrounds for the analysis are $e e \rightarrow e e$ and $e e \rightarrow e \gamma$ events, making the identification of very forward electrons and photons crucial. The results would improve the last LEP data by about a factor 10: $\Lambda>6.33 \mathrm{TeV}$ at CLIC compared to $\Lambda>431 \mathrm{GeV}$ at LEP [8].

The vector boson scattering process [9] provides a test of the electroweak symmetry breaking mechanism and is sensitive to possible new physics in the Higgs sector. The measurement of anomalous quartic gauge boson couplings ( 0 in case of SM) or the observation of additional resonances would be clear signs of new physics. For the results presented here, the analysis is performed at generator level with only information on the separation between hadronic $W$ and $Z$ decays taken from full simulation. Figure 3 shows the precision on the measurement of the anomalous couplings, $F_{S 0}, F_{S 1}$, for different centre-of-mass energy and polarisation scenario. It is evident that increasing the centre-of-mass energy largely improves the sensitivity of the analysis: the gain is of about a factor 10 going from $1 \mathrm{TeV}$ to $3 \mathrm{TeV}$. Assuming $80 \%$ polarisation of the electron beam improves the precision further by about a factor of 1.5 . For $3 \mathrm{TeV}$ the final precision on the couplings is $\Delta F_{S 0,1} \sim 5 \mathrm{TeV}^{-4}$, about 100 times better than the $8 \mathrm{TeV}$ results at the LHC experiments $[10,11]$. Therefore, given the great potential of this channel at CLIC, studies are now ongoing using full simulation. 


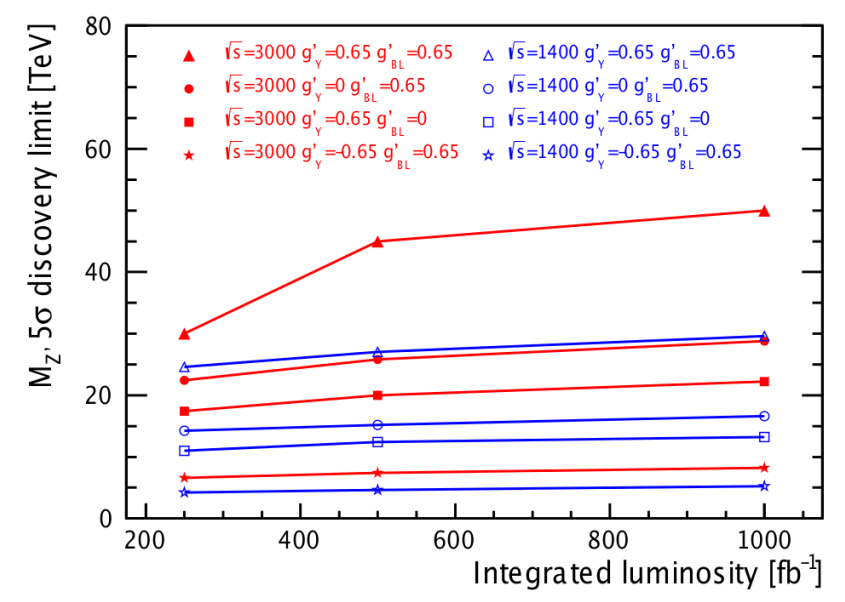

Figure 4: Discovery potential as a function of the integrated luminosity for different values of the couplings $g_{Y}^{\prime}$ and $g_{B L}^{\prime}$ at $3 \mathrm{TeV}$ (red) and $1.4 \mathrm{TeV}$ (blue) [12].

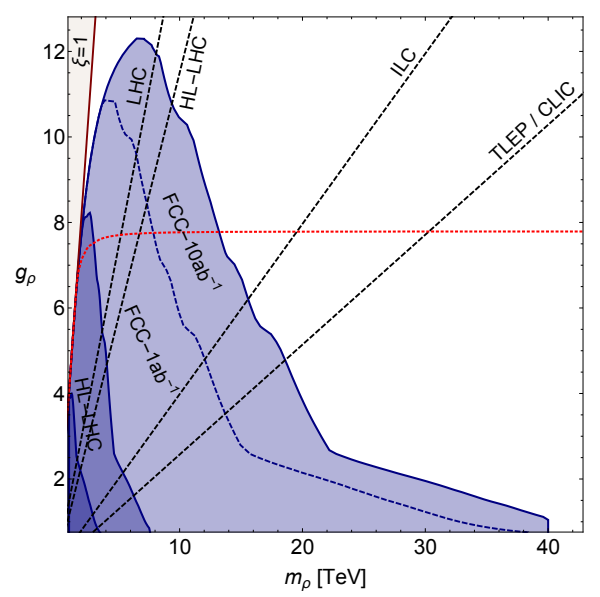

Figure 5: Comparison of direct and indirect searches in the $\left(m_{\rho}, g_{\rho}\right)$ plane for LHC, HL-LHC, FCC, ILC and CLIC [13].

Many SM extensions predict additional $U^{\prime}(1)$ gauge symmetries with associated $Z^{\prime}$ gauge bosons. The minimal anomaly-free $Z^{\prime}$ scenario predicts no extra exotic fermions in the particle spectrum and only 3 extra free parameters: the $Z^{\prime}$ mass and two effective couplings, $g_{Y}^{\prime}$ and $g_{B L}^{\prime}$. Prospects for constraining $Z^{\prime}$ models at CLIC were studied for the $e e \rightarrow \mu \mu$ process [12]. The discovery potential and the coupling determination are based on the measurements of three observables: the total cross section for the process, the forward-backward asymmetry and the left-right asymmetry. The first two observables are measured with no beam polarisation, while the third is computed using $\pm 80 \%$ polarisation for the electron beam. The results are shown in Figure 4 as a function of the integrated luminosity: limits up to tens of $\mathrm{TeV}$ can be set. It is interesting to notice that increasing the centre-of-mass energy would improve the analysis sensitivity more than increasing the integrated luminosity. This is due to the systematic uncertainties becoming dominant over the statistical uncertainty for $\mathscr{L}>500 \mathrm{fb}^{-1}$. Finally, if a $Z^{\prime}$ is discovered, either at LHC or CLIC itself, CLIC will be able to provide precise measurements of its properties.

Some BSM scenario, like the minimal Composite Higgs, predict corrections to the SM Higgs couplings [13]. CLIC could provide high precision measurements of such couplings [14]: at O(1\%) in a model independent evaluation or even at $\mathrm{O}(0.1 \%)$ assuming the same hypotheses as for the LHC analyses. Beyond indirect effects, the minimal Composite Higgs scenario also predicts direct signatures like electroweak (EW) vector resonances. These can be described by a simple model with only two parameters: the resonance mass, $m_{\rho}$, and the intrinsic coupling between SM fermions and EW gauge bosons, $g_{\rho}$ [13]. Figure 5 shows the complementarity of direct and indirect searches between hadron and lepton colliders. In the $\left(m_{\rho}, g_{\rho}\right)$ plane the modification of the Higgs couplings translates in the exclusion of the area above the dashed lines in the figure. It is evident that a possible future collider like FCC-hh could give direct access to high mass narrow resonances, thanks to the very high centre-of-mass energy, on the other hand these limits are not very stringent for large values of the effective coupling. Instead the precision offered by lepton colliders, and by CLIC in particular, would be able to test the high coupling region. 


\section{Conclusion}

CLIC is an attractive option for the future of CERN. The clean environment and the low level of background provided by lepton collisions combined with the high centre-of-mass energy gives a great potential for BSM searches: both for direct production and indirect precision measurements. Through a series of physics benchmark studies in full simulation, it has been proven that in many channels CLIC could extend and complement the results from past and current colliders, LEP and LHC. Moreover, if any new particle is discovered at the LHC or CLIC itself, CLIC could provide precision measurements of its properties. More channels and final states are currently under study to fully probe the CLIC potential for BSM physics.

\section{References}

[1] M. J. Boland et al. [CLIC and CLICdp Collaborations], Updated baseline for a staged Compact Linear Collider, doi:10.5170/CERN-2016-004 arXiv: 1608.07537 [physics.acc-ph].

[2] T. Abe et al., ILD Concept Group, The International Large Detector: Letter of Intent, DESY-2009-87. FERMILAB-PUB-09-682-E. KEK-REPORT-2009-6 (2010), arXiv: 1006.3396 [hep-ex].

[3] H. Aihara et al., SiD Letter of Intent (2009), arXiv: 0911.0006 [physics.ins-det].

[4] T. Behnke et al., The International Linear Collider Technical Design Report - Volume 1: Executive Summary (2013), arXiv: 1306.6327 [physics.acc-ph].

[5] L. Linssen et al., CLIC Conceptual Design Report: Physics and Detectors at CLIC, CERN-2012-003. ANL-HEP-TR-12-01. DESY-12-008. KEK-REPORT-2011-7 (2012), arXiv: 1202.5940 [physics.ins-det].

[6] H. Abramowicz et al. [CLICdp Collaboration], Physics at the CLIC $e^{+} e^{-}$Linear Collider - Input to the Snowmass process 2013, arXiv:1307.5288 [hep-ex].

[7] I. Boyko and Y. Nefedov, Study of ee $\rightarrow \gamma \gamma$, CLIC workshop 2016, https://indico.cern.ch/event/449801/contributions/1945364/attachments/1213550/1770931/boyko_eegg.pdf.

[8] S. Schael et al. [ALEPH and DELPHI and L3 and OPAL and LEP Electroweak Collaborations], Electroweak Measurements in Electron-Positron Collisions at W-Boson-Pair Energies at LEP, Phys. Rept. 532 (2013) 119 doi:10.1016/j.physrep.2013.07.004, arXiv:1302.3415 [hep-ex].

[9] C. Fleper, W. Kilian, J. Reuter and M. Sekulla, Scattering of $W$ and $Z$ Bosons at High-Energy Lepton Colliders, DESY 16-098. SI-HEP-2016-21. KA-TP-11-2016, arXiv:1607.03030 [hep-ph].

[10] The ATLAS collaboration, Evidence of the electroweak production of WWjj in pp collisions at $8 \mathrm{TeV}$ with the ATLAS detector, ATLAS-CONF-2014-013.

[11] The CMS collaboration, Evidence for exclusive gamma-gamma to WW production and constraints on Anomalous Quartic Gauge Couplings at 8 TeV, CMS-PAS-FSQ-13-008.

[12] J. J. Blaising and J. D. Wells, Physics performances for Z' searches at $3 \mathrm{TeV}$ and $1.5 \mathrm{TeV} C L I C$, arXiv:1208.1148 [hep-ph].

[13] A. Thamm, R. Torre and A. Wulzer, Future tests of Higgs compositeness: direct vs indirect, JHEP 1507 (2015) 100 doi:10.1007/JHEP07(2015)100, arXiv: 1502.01701 [hep-ph].

[14] H. Abramowicz et al. [CLICdp Collaboration], Higgs Physics at the CLIC Electron-Positron Linear Collider, arXiv:1608.07538 [hep-ex]. 